\title{
Changes in serum thymidine kinase 1 levels during chemotherapy correlate with objective response in patients with advanced gastric cancer
}

\author{
YONGPING LIU ${ }^{1,2^{*}}$, YANG LING ${ }^{2 *}$, QIUFENG QI ${ }^{1}$, YEXIN TANG ${ }^{1}$, JIANZHONG XU $^{2}$, \\ TONG ZHOU ${ }^{2}$, GUIFENG SHENG ${ }^{2}$, QUANLIANG YANG ${ }^{2}$ and YAODONG PAN ${ }^{3}$ \\ ${ }^{1}$ Clinical Oncology Laboratory, and Departments of ${ }^{2}$ Oncology, ${ }^{3}$ Gastroenterological Surgery, \\ Changzhou Tumor Hospital Affiliated with Suzhou University, Changzhou 213002, P.R. China
}

Received July 4, 2011; Accepted August 2, 2011

DOI: $10.3892 /$ etm.2011.338

\begin{abstract}
Serum thymidine kinase 1 (STK1) is a reliable proliferation marker in most solid tumors, including gastric cancer. The aim of this study was to evaluate whether STK1 levels are related to the tumor response to chemotherapy and survival in advanced gastric cancer. The results showed that the average STK1 level in patients with gastric cancer $(5.57 \pm 3.07 \mathrm{pM})$ was significantly higher than that in the healthy controls $(1.12 \pm 0.57)$ $(\mathrm{P}<0.001)$. Among the 84 patients, the average STK1 level $(6.02 \pm 3.12)$ in the 56 patients who did not undergo surgery was higher than the level $(4.68 \pm 2.78)$ in the 28 patients who received surgery $(\mathrm{P}=0.049)$. The STK1 value correlated with clinical stage, ECOG PS and serum CEA levels $(\mathrm{P}<0.001, \mathrm{P}=0.001$ and $\mathrm{P}=0.004$, respectively), but not with age and gender. The average STK1 levels after 1,2 and 4 cycles of chemotherapy did not significantly decrease in the total patients, when compared to the levels prior to chemotherapy. Yet, after 2 cycles of chemotherapy, the average level of STK1 was significantly decreased in patients who achieved an objective response (OR) $(C R, P R$ or no recurrence). Particularly after 1 cycle of chemotherapy, the average level of STK1 in patients who achieved OR started to decline, while in most of the patients with disease progression or recurrence, the STK1 level started to increase. In patients receiving palliative chemotherapy or receiving adjuvant chemotherapy, a significant difference in the median PFS (median PFS, not defined vs. 4 months, $\mathrm{P}<0.001$ ) or RFS (median RFS, not defined vs. 5 months, $\mathrm{P}<0.001$ ) was noted between patients with decreased STK1 levels and patients with increased STK1
\end{abstract}

Correspondence to: Dr Yongping Liu, Clinical Oncology Laboratory, Changzhou Tumor Hospital Affiliated with Suzhou University, North Huaide Road 1, Changzhou 213002, P.R. China

E-mail: liuyongping026@yahoo.com.cn

${ }^{*}$ Contributed equally

Key words: tumor marker, chemotherapy, objective response, survival, gastric cancer levels during the first 2 months of chemotherapy. The log-rank test showed that patients with decreased STK1 levels had a trend of a longer OS in the palliative chemotherapy group. Our results suggest that serum TK1 levels correlate with clinical stage, ECOG PS and serum CEA levels in patients with gastric cancer, and changes in STK1 levels during the first 2 months of chemotherapy may be more important for evaluating chemotherapy response, predicting PFS and RFS than baseline values of STK1 in patients with advanced gastric cancer.

\section{Introduction}

Despite its declining incidence, gastric cancer is still the second most common cause of death from cancer in Asia and worldwide $(1,2)$. Even after radical surgery, the majority of gastric cancer patients develop local or distant recurrence (3). Recently, combination chemotherapy has become the standard of care for patients with advanced gastric cancer, as it has been proven effective for improving the survival and quality of life of these patients (4). However, the average overall benefit of chemotherapy is small, and the majority of patients with advanced gastric cancer still do not appear to draw any meaningful benefit from chemotherapy. An objective response (OR) to chemotherapy is the primary study endpoint and one of the surrogate markers of clinical benefit. Therefore, monitoring OR to chemotherapy is essential for assessing prognosis and planning further treatment. Computed tomography (CT) scans are often adopted by physicians to evaluate the objective tumor response to chemotherapy. Due to the high cost and adverse effects of radioactivity, CT scans are used by physicians to evaluate the objective tumor response to chemotherapy usually after 2 or 3 cycles of chemotherapy. However, approximately $50 \%$ of patients with advanced gastric cancer do not benefit from any one of the chemotherapy regimens during the first cycle of chemotherapy. The early identification of no-response patients is very difficult for most physicians. Thus, the development of more straighforward, less costly and safer tools with which to monitor the effects of chemotherapy in patients with gastric cancer would be extremely valuable. Serum tumor markers may be used for this purpose. Recently, among the available serum markers, TK1 has been the most widely 
tested in solid tumors, including gastric cancer, particularly for monitoring the effect of tumor therapies, prognosis and follow-up. Yet, its role as a marker of treatment efficacy is still contradictory to date.

The aim of the present study was to investigate the role of serum TK1 during chemotherapy treatment as an adjuvant or surrogate marker for monitoring the effects of therapy and prognosis in patients with advanced gastric cancer who received chemotherapy.

\section{Patients and methods}

In total, 84 advanced gastric cancer patients previously untreated with chemotherapy were enrolled prospectively into this single-institution study from September 2009 to May 2010. These patients were pathologically diagnosed with gastric adenocarcinoma by endoscopic biopsy or surgical tumor specimens. Among these patients, 56 patients presenting with at least one measurable lesion received palliative chemotherapy, and 28 patients received adjuvant chemotherapy after surgery. All patients were treated with FOLFOX4 or DCF regimens. FOLFOX4 consisted of L-HOP $85 \mathrm{mg} / \mathrm{m}^{2}$ intravenously (i.v.) only on Day 1, with leucovorin $100 \mathrm{mg} / \mathrm{m}^{2}$ i.v. as a 2-h infusion followed by bolus 5 -fluorouracil (5-FU) $400 \mathrm{mg} / \mathrm{m}^{2} /$ day and a 22 -h infusion of $5-\mathrm{FU} 600 \mathrm{mg} / \mathrm{m}^{2} / \mathrm{day}$, repeated for two consecutive days every 2 weeks for a minimum of 6 cycles. DCF regimen was administered according to the following plan using docetaxel at a dose of $20 \mathrm{mg} / \mathrm{m}^{2}$ i.v. administered over $30 \mathrm{~min}$ on Day 1, with cisplatin at a dose of $20 \mathrm{mg} / \mathrm{m}^{2}$ administered over $1 \mathrm{~h}$ on Day 2, and 5 -FU $350 \mathrm{mg} / \mathrm{m}^{2} /$ day, repeated for 5 consecutive days every 3 weeks for a minimum of 3 cycles. Written informed consent was obtained from the patients for chemotherapy, and data regarding the patient clinical and pathological features were collected for this study.

Analysis of remission. For patients with measurable lesions, the tumor response was assessed according to Response Evaluation Criteria in Solid Tumors (RECIST) criteria (5). Accordingly, a complete response (CR) was defined as the disappearance of all target lesions. Any pathological lymph nodes (whether target or non-target) were required to show a reduction in the short axis to $<10 \mathrm{~mm}$. A partial response (PR) was defined as at least a $30 \%$ decrease in the sum of the diameters of the target lesions, taking as reference the baseline sum diameters. Progressive disease (PD) was defined as at least a $20 \%$ increase in the sum of the diameters of target lesions, taking as reference the smallest sum during study. In addition to the relative increase of $20 \%$, the sum must also demonstrate an absolute increase of at least $5 \mathrm{~mm}$. Stable disease (SD) was defined as neither sufficient shrinkage to qualify for PR nor sufficient increase to qualify for PD, taking as reference the smallest sum of diameters during study. In this study, patients who achieved a CR or PR were classified as having a chemotherapeutic objective response (OR), and all remaining patients were considered as non-responders. For postoperative patients with no measurable lesions, a chemotherapeutic OR was defined as no discovery of all objective evidence of new disease, and non-response was defined as discovery of new disease by CT scan or pathological examination.
Follow-up. Interim history, physical examination, hematologic studies, serum thymidine kinase 1 (STK1) levels, carcinoembryonic antigen (CEA) levels were assessed every 1 month during treatment, and whole-body CT was performed every 2 months during treatment. The above tests were carried out every 3 months in the first year and every 6 months thereafter. Patients underwent upper endoscopy every 2 months during treatment and every 6 months thereafter. The progression or recurrence of gastric carcinoma was confirmed by cytology biopsy, surgery or whole-body CT. The 7th edition of the UICC TNM staging system for gastric cancer was used for the classification of each case. The study was carried out in a blinded manner so that patient outcome was unknown to the investigators performing the molecular analyses. Progression-free survival (PFS) was the time from study entry until disease progression or death or the day of the last follow-up visit whichever came first. Relapse-free survival (RFS) was the time from study entry until recurrence or death to the day of the last follow-up visit whichever came first. Overall survival (OS) was the time from the day of the confirmation of diagnosis until the date of death regardless of cause or the most recent documented follow-up.

Detection of serum TK1. Serum samples were obtained immediately before the start of chemotherapy and every 1 month during the chemotherapy break. STK1 was analyzed by an ECL dot blot assay. The procedure was performed according to the manufacturer's protocol (commercial kit; SSTK, Shenzhen, China) as described elsewhere (6).

Statistical methods. Differences in the values among the groups under study were evaluated by analysis using the independent-samples t-test, as indicated. The paired-samples t-test procedure was used to compare STK1 values prior to treatment and after chemotherapy. A P-value $\leq 0.05$ was indicative of statistical significance.

\section{Results}

STK1 levels of the healthy control and patients prior to chemotherapy. The average STK1 level in all of the patients with gastric cancer was $5.57 \pm 3.07 \mathrm{pM}$, which was significantly higher than that in the healthy controls $(1.12 \pm 0.57 \mathrm{pM})$ $(\mathrm{P}<0.001)$. The average STK1 value in patients who did not receive surgery $(6.02 \pm 3.12)$ was significantly higher than that in patients who received surgery $(4.68 \pm 2.78)(\mathrm{P}<0.05)$, which may have resulted from the decrease in tumor volume burden.

Associations between STK1 levels and clinicopathological features. There was a significant increase in the average STK1 value from stage I + II $(2.26 \pm 1.09)$ to stage III + IV $(6.28 \pm 4.23)$ disease $(\mathrm{P}<0.001)$. The STK1 value was increased by 1.78 times in patients with distant metastasis compared with patients without metastasis. A high STK1 value was also correlated with a poor Eastern Cooperative Oncology Group performance status (ECOG $\mathrm{PS})(\mathrm{P}=0.001)$ and high serum CEA levels $(\mathrm{P}=0.004)$, but not with age and gender (Table I).

Associations between STK1 levels and remission in patients treated with palliative chemotherapy. In all of the 56 patients who received palliative chemotherapy, the mean STK1 
Table I. Associations between STK1 levels and clinicopathological features of the patients.

\begin{tabular}{lccc}
\hline Type & $\mathrm{n}$ & Mean $\pm \mathrm{SD}$ & P-value \\
\hline $\begin{array}{l}\text { Age }{ }^{\mathrm{a}} \text { (years) } \\
\leq 60\end{array}$ & 43 & $5.03 \pm 3.22$ & \\
$>60$ & 41 & $5.92 \pm 4.92$ & 0.327 \\
Gender & & & \\
$\quad$ Male & 62 & $7.12 \pm 6.24$ & \\
Female & 22 & $4.88 \pm 2.92$ & 0.116 \\
CEA & & & \\
$\geq 5 \mathrm{ng} / \mathrm{ml}$ & 28 & $7.41 \pm 4.32$ & \\
$<5 \mathrm{ng} / \mathrm{ml}$ & 56 & $4.49 \pm 3.71$ & 0.004 \\
Stage & & & \\
I + II & 17 & $2.26 \pm 1.09$ & \\
III + IV & 67 & $6.28 \pm 4.23$ & $<0.001$ \\
ECOG PS & & & \\
0,1 & 56 & $4.13 \pm 2.60$ & \\
2 & 28 & $8.14 \pm 5.26$ & 0.001
\end{tabular}

${ }^{a}$ Median age of the patients was 60 years. CEA, carcinoembryonic antigen; ECOG PS, Eastern Cooperative Oncology Group performance status.

value before the start of treatment was $6.02 \mathrm{pM}$, which was not significantly different from the values after 2 months of chemotherapy. The mean STK1 value prior to chemotherapy in the CR patients $(n=4)$ was $6.82 \mathrm{pM}$, the PR patients $(\mathrm{n}=23)$ $5.84 \mathrm{pM}$, the SD patients $(\mathrm{n}=10) 6.55 \mathrm{pM}$ and the PD patients $(\mathrm{n}=19) 6.62 \mathrm{pM}$ (no significant difference was noted between any two groups). The corresponding STK1 values decreased significantly after 2 and 4 months of treatment in the patients who obtained $\mathrm{CR}(\mathrm{P}=0.025$ and $\mathrm{P}=0.043$, respectively) and $\mathrm{PR}$ $(\mathrm{P}<0.001$ and $\mathrm{P}=0.001$, respectively), while in the patients who obtained PD, the corresponding STK1 value increased significantly after 2 months of the treatment $(\mathrm{P}=0.004)$ (Fig. 1). After 1 month of chemotherapy, the corresponding STK1 values started to decline in the OR groups $(\mathrm{P}=0.035$ in $\mathrm{CR}$ group and $\mathrm{P}<0.001$ in $\mathrm{PR}$ group) but increased in the $\mathrm{PD}$ group $(\mathrm{P}=0.011)$, respectively. As the patients with PD during the first 2 months of treatment received other treatment regimens, we did not compare the difference between the STK1 value prior to chemotherapy and that after 4 months of treatment. Compared with the value prior to chemotherapy, the mean STK1 value after 4 months of chemotherapy did not decrease significantly in patients with $\mathrm{SD}$.

Associations between STK1 levels and recurrence in patients treated with surgery following by chemotherapy. In all 28 patients who received surgery followed by adjuvant chemotherapy, the mean STK1 value prior to chemotherapy was $4.68 \mathrm{pM}$, which was not significantly different from the value after 2 months of chemotherapy. The mean STK1 values before the start of chemotherapy were not correlated with clinical response. After 2 and 4 months of chemotherapy, the

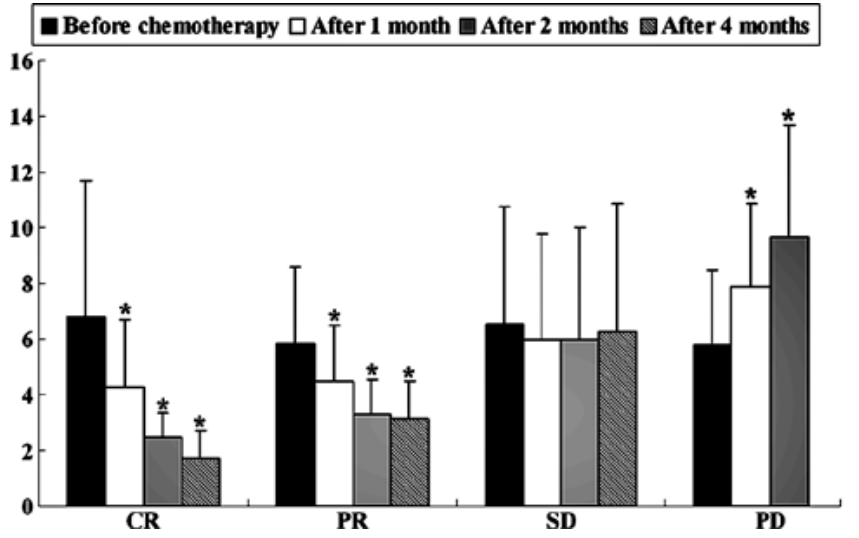

Figure 1. Associations between changes in STK1 concentrations and remission in gastric cancer patients treated with palliative chemotherapy. CR, complete remission; PR, partial remission; SD, stable disease; PD, progressive disease. ${ }^{*} \mathrm{P}<0.05$ vs. the STK1 values prior to chemotherapy.

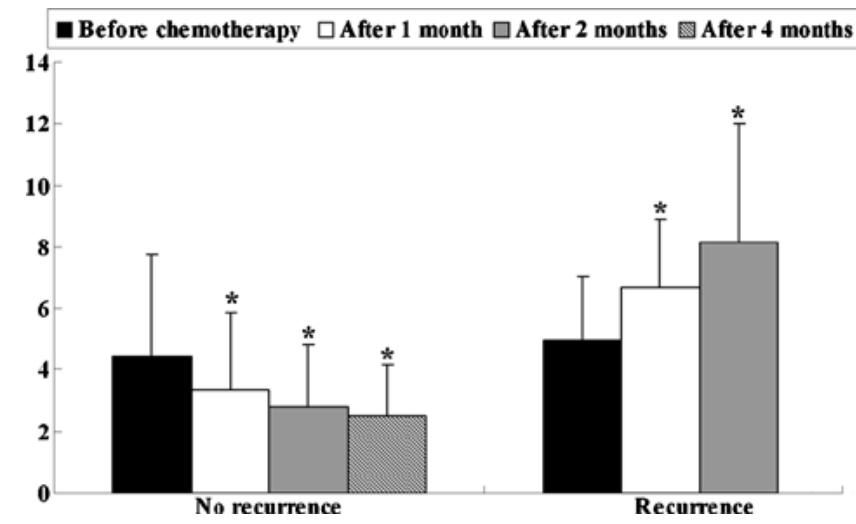

Figure 2. Associations between changes in STK1 concentrations and recurrence in gastric cancer patients treated with adjuvant chemotherapy. ${ }^{*} \mathrm{P}<0.05$ vs. the STK1 values prior to chemotherapy.

STK1 values decreased significantly in the patients without recurrence $(\mathrm{P}=0.011$ and $\mathrm{P}=0.002$, respectively), while in patients with recurrence, the STK1 values increased significantly $(\mathrm{P}=0.003)$ after 2 months of chemotherapy, compared with that before chemotherapy (Fig. 2). Particularly, after 1 month of chemotherapy, the corresponding STK1 values started to decline in the patients without recurrence $(\mathrm{P}=0.043)$ and to increase in the recurrence group $(\mathrm{P}=0.003)$. As patients with recurrence during the first 2 months of treatment received other treatment regimens, we did not compare the difference between the STK1 value before chemotherapy and that after 4 months of treatment. Compared with the value before chemotherapy, the mean STK1 value after 4 months of chemotherapy did not decrease significantly in patients with SD.

Associations between STK1 levels and survival. The median progression-free survival of all patients was 9 months (range, 2-17 months), and due to the short follow-up period, the median overall survival time could not been defined. In the patient group receiving palliative chemotherapy, patients with decreased STK1 levels during the first 2 months of treatment had significantly longer median PFS than those with increased STK1 levels for the same treatment interval (median PFS, not 
A

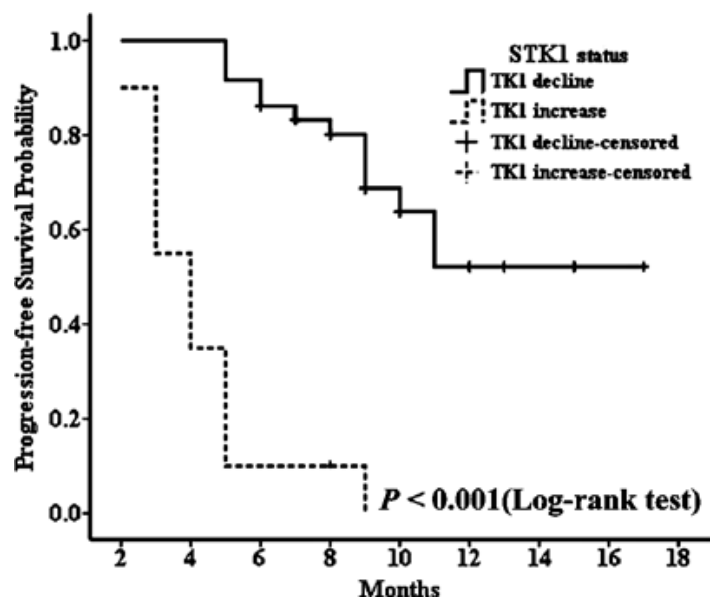

B

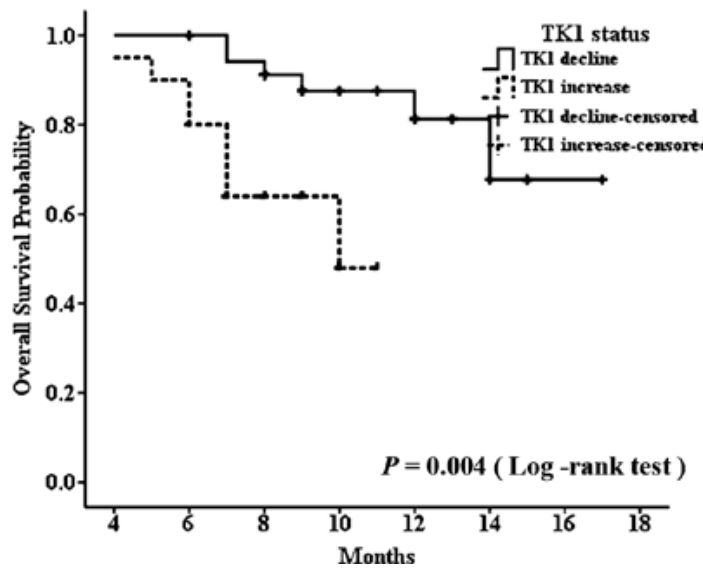

Figure 3. Progression-free survival curve (A) and overall survival curve (B) for patients treated with palliative chemotherapy, according to decreased and increased levels of STK1.

defined vs. 4 months, $\mathrm{P}<0.001$ ) (Fig. 3A). At the same time, we found similar results in the group receiving adjuvant chemotherapy; patients with decreased STK1 levels during the first 2 months of treatment had significantly longer median RFS than those with increased STK1 levels for the same time period (median RFS, not defined vs. 5 months, $\mathrm{P}<0.001$ ) (Fig. 4). Although we could not determine the median overall survival time of the patients with decreased or increased STK1 levels in both groups, the log-rank test showed that patients receiving palliative chemotherapy with decreased STK1 levels during the first 2 months of treatment had longer OS (Fig. 3B). Since only 2 patients died, a meaningful determination of the association between the STK1 level and OS was not possible in the adjuvant chemotherapy patients. Yet, our results indicate that a change in STK1 levels during the first 2 months of chemotherapy predicts the PFS in patients receiving palliative chemotherapy or RFS in patients receiving adjuvant chemotherapy, which may aid clinicians to timely select new treatment regimens for patients who do not benefit from firstline chemotherapy regimens.

\section{Discussion}

The purpose of cancer chemotherapy is to improve the overall survival of patients. An objective response (OR) to

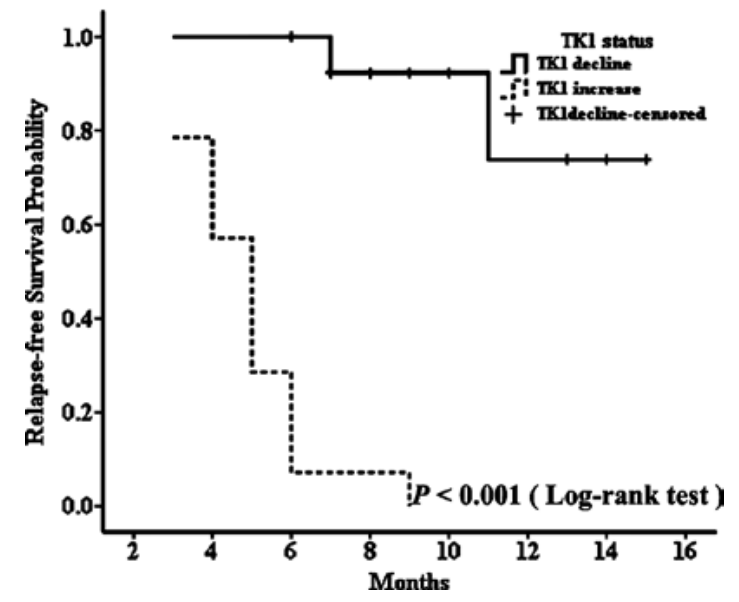

Figure 4. Relapse-free survival curve for patients treated with adjuvant chemotherapy, according to decreased and increased levels of STK1.

chemotherapy often serves as a surrogate marker of clinical benefit, as it is associated with a better survival outcome (7). In clinical settings, most oncologists have adopted the use of repeated imaging studies to evaluate tumor response and make decisions concerning continued therapy on the basis of both objective and symptomatic criteria. Even for patients who undergo sugery yet still require adjuvant chemotherapy, many clinicians follow up their patients' malignant disease by means of repeated imaging analysis to evaluate whether or not the disease has progressed. Although evaluation criteria based on imaging modalities, including CT, have become standard in the evaluation of tumor response, serum levels of tumor markers are sometimes used in clinical settings as adjuvant or surrogate markers for estimation of tumor response, particularly in patients with no measurable lesions.

TK1 is a pyrimidine salvage pathway enzyme involved in DNA synthesis and repair. TK1 activity is low or absent in resting cells, starts to increase in the late G1 stage, increases in S-phase, and disappears during mitosis (8). The G2/M specific TK1 activity disappearance is due to specific degradation of TK1 protein by the ubiquitin-proteasome pathway (8). The activity or concentration of TK1 is correlated closely with cell proliferation of cultured cell lines or tissues (9). It has been reported that TK1 levels in the serum of patients with malignancies are significantly higher than those of healthy persons (10), due to the fact that most cancer cells die in the G2/S phase of the cell cycle while normal cells often die in G1. In the present study, the average STK1 level in all of the patients with gastric cancer was $5.57 \pm 3.07 \mathrm{pM}$, while that in the healthy controls was $1.12 \pm 0.57 \mathrm{pM}(\mathrm{P}<0.001)$. Our results coincided with the above results (11). Base on our results and those of other studies, we can speculate that STK1 may be an additional biomarker for cancer diagnosis.

The high levels of STK1 in patients with malignancies also suggest that TK1 may be a useful proliferation marker for the assessment of tumor progression or for monitoring therapy. He et al (11) and Zou et al (12) reported that the STK1 activity was elevated in patients before surgery, but was irregular following surgery and adjuvant treatment. Chen et al (13) reported that the mean STK1 values decreased significantly after treatments (surgery and/or chemotherapy, $\mathrm{P}<0.01$ ) in 
4 types of cancers, apart from patients with gastric cancer In our 56 gastric cancer patients who received only chemotherapy, the mean STK1 value before the start of treatment was not significantly different from the values after 2 months of chemotherapy. Even in the 28 patients who received surgery followed by adjuvant chemotherapy, the mean STK1 value prior to chemotherapy was not significantly different from the values after chemotherapy. Yet, in patients who obtained CR, PR and no recurrence, the STK1 values declined significantly after 2 months of treatment $(\mathrm{P}=0.025, \mathrm{P}<0.001$ and $\mathrm{P}=0.011$, respectively), while in patients who obtained $\mathrm{PD}$, the corresponding STK1 values increased significantly $(\mathrm{P}=0.004$ and $\mathrm{P}=0.003$, respectively) (Figs. 1 and 2 ). In both groups, the mean STK1 values before the start of chemotherapy were not correlated to clinical response. Our results were partially in accordance with those of Chen et al (13) and He et al (14). Our results suggest that the change in STK1 levels during the first 2 months of treatment may be more important than baseline levels of STK1 in predicting an individual's chemotherapy response. Thus, to determine changes in STK1, it is necessary for clinicians to establish a baseline level of the STK1 concentration for every patient. In the present study, after 1 cycle of chemotherapy, the levels of STK1 in patients with PD started to increase, which suggests that clinicians should pay special attention to patients with elevated levels of STK1 during the first adjuvant/palliative chemotherapy break. In the event a patient with an increase in STK1 also presents with new emerging clinical symptoms or other elevated tumor markers, the clinician should adjust the treatment regimen for this patient early in time.

Many studies have shown that increased serum TK1 levels adversely affect OS (15-18). In our study, because of the short follow-up period as most of the patients were followed up for less than 2 years, a meaningful determination of the association between STK1 level and overall survival was not possible. Yet, a significant difference in the median PFS (median PFS, not defined vs. 4 months, $\mathrm{P}<0.001$ ) or median RFS (median RFS, not defined vs. 5 months, $\mathrm{P}<0.001$ ) was noted between patients with decreased STK1 levels and patients with increased STK1 levels during the first 2 months of treatment. Our results suggest that changes in STK1 during the first 2 months of chemotherapy may be more valuable for evaluating tumor chemotherapy response, predicting PFS and RFS than baseline values of STK1 in gastric cancer patients who receive chemotherapy.

We also investigated the association of STK1 with clinicopathologic characteristics. A significant association was observed between STK1 values and clinical stage, and there was a significant increase in the average STK1 value from stage I + II $(2.26 \pm 1.09)$ to stage III + IV $(6.28 \pm 4.23)(\mathrm{P}<0.001)$. It appeared that patients with a large tumor burden possess more potential for cell proliferation in gastric cancer. In our study, a high STK1 value was also correlated with a poor ECOG PS $(\mathrm{P}=0.001)$ and high serum CEA levels $(\mathrm{P}=0.004)$, but not with age and gender (Table I). These results partially coincide with those of Chen et al (13).

The limitations of the present study included the relatively small size of the sample and the short follow-up period, which may explain the weak prognostic value of STK1 for overall survival in our study. To further confirm the prognostic value of STK1 in gastric cancer patients who receive chemotherapy, a larger sample and longer period of follow-up is needed in future studies.

\section{Acknowledgements}

The research was supported, in part, by the Science and Technology Planning Project of Changzhou (CS20092025) and the Key Medical Innovation Talents Training Project of Changzhou, Jiangsu Province, China.

\section{References}

1. Leung WK, Wu MS, Kakugawa Y, et al: Screening for gastric cancer in Asia: current evidence and practice. Lancet Oncol 9: 279-287, 2008

2. Kamangar F, Dores GM and Anderson WF: Patterns of cancer incidence, mortality, and prevalence across five continents: defining priorities to reduce cancer disparities in different geographic regions of the world. J Clin Oncol 24: 2137-2150, 2006

3. Macdonald JS: Treatment of localized gastric cancer. Semin Oncol 31: 566-573, 2004

4. Wagner AD, Unverzagt S, Grothe W, et al: Chemotherapy for advanced gastric cancer. Cochrane Database Syst Rev CD004064, 2010.

5. Eisenhauer EA, Therasse P, Bogaerts J, et al: New response evaluation criteria in solid tumours: revised RECIST guideline (version 1.1). Eur J Cancer 45: 228-247, 2009.

6. Xu XH, Zhang YM, Shu XH, et al: Serum thymidine kinase 1 reflects the progression of pre-malignant and malignant tumors during therapy. Mol Med Rep 1: 705-711, 2008.

7. Paesmans M, Sculier JP, Libert P, et al: Response to chemotherapy has predictive value for further survival of patients with advanced non-small cell lung cancer: 10 years experience of the European Lung Cancer Working Party. Eur J Cancer 33: 2326-2332, 1997.

8. Ke PY and Chang ZF: Mitotic degradation of human thymidine kinase 1 is dependent on the anaphase-promoting complex/ cyclosome-CDH1-mediated pathway. Mol Cell Biol 24: 514-526, 2004.

9. He Q, Mao Y, Wu J, et al: Cytosolic thymidine kinase is a specific histopathologic tumour marker for breast carcinomas. Int J Oncol 25: $945-953,2004$

10. O'Neill KL, Buckwalter MR and Murray BK: Thymidine kinase: diagnostic and prognostic potential. Expert Rev Mol Diagn 1: 428-433, 2001

11. He Q, Zou L, Zhang PA, Lui JX, Skog S and Fornander T: The clinical significance of thymidine kinase 1 measurement in serum of breast cancer patients using anti-TK1 antibody. Int J Biol Markers 15: 139-146, 2000.

12. Zou L,Zhang PG,Zou S, Li Y and He Q: The half-life of thymidine kinase 1 in serum measured by ECL dot blot: a potential marker for monitoring the response to surgery of patients with gastric cancer. Int J Biol Markers 17: 135-140, 2002.

13. Chen Y, Ying M, Hu M, et al: Serum thymidine kinase 1 correlates to clinical stages and clinical reactions and monitors the outcome of therapy of 1,247 cancer patients in routine clinical settings. Int J Clin Oncol 15: 359-368, 2010.

14. He Q, Fornander T, Johansson H, et al: Thymidine kinase 1 in serum predicts increased risk of distant or loco-regional recurrence following surgery in patients with early breast cancer. Anticancer Res 26: 4753-4759, 2006

15. Kallander CF, Simonsson B, Hagberg H and Gronowitz JS: Serum deoxythymidine kinase gives prognostic information in chronic lymphocytic leukemia. Cancer 54: 2450-2455, 1984.

16. Hallek M, Langenmayer I, Nerl C, et al: Elevated serum thymidine kinase levels identify a subgroup at high risk of disease progression in early, nonsmoldering chronic lymphocytic leukemia. Blood 93: 1732-1737, 1999.

17. Hallek M, Wanders L, Ostwald M, et al: Serum beta(2)-microglobulin and serum thymidine kinase are independent predictors of progression-free survival in chronic lymphocytic leukemia and immunocytoma. Leuk Lymphoma 22: 439-447, 1996.

18. Magnac C, Porcher R, Davi F, et al: Predictive value of serum thymidine kinase level for Ig-V mutational status in B-CLL. Leukemia 17: 133-137, 2003. 
